247. G. Peach $\quad 1970, J$. Phys. B, 3, 328.

248. G. Peach 1971, J. Phys. B, 4, 1670.

249. D. L. Moores 1972, J. Phys. B, 5, 286.

250. K. L. Aitken $1971, J$. Phys. B, 4, 1176 and 1189.

251. E. Stingl 1972, J. Phys. B, 5, 1160.

252. J. B. Hasted, G. L. Awad 1972, J. Phys. B, 5, 1719.

253. H. J. Kunze 1971, Phys Rev. A, 3, 937.

254. B. Steiner 1972, see ref. (81) p. 483.

255. B. Peart, D. S. Walton, K. T. Dolder $1970, J$. Phys. B, 3, 1346.

256. D. S. Walton, B. Peart, K. T. Dolder 1971, J. Phys. B, 4, 1343.

257. F. H. Faisal, A. K. Bathia 1972, Phys Rev., 5, A2144.

258. A. H. Gabriel, T. M. Paget 1972, J. Phys. B, 5, 673.

259. H. P. Summers 1972, Mon. Not. R. Astron. Soc., 158, 255.

260. M. Blaha 1972, Astro. Let., 10, 179.

HENRI VAN REGEMORTER

Chairman of the Committee.

\title{
COMMITTEE 4: STRUCTURE OF ATOMIC SPECTRA
}

An excellent review of the present state of the analysis and of future trends has been prepared by B. Edlén (10). He discusses the electronic structure of the ground configurations, "arranged in order of the total number of electrons, $N$, for the first half of the periodic system". "The intervals within the ground-configurations are of particular astrophysical significance. The $Z$-dependence of these intervals can be expressed with remarkable accuracy by quite simple relations." He points out that the electrostatic term intervals, which account for the forbidden nebular lines, have a linear $Z$-dependence, while the spin-orbit intervals, increasing with the fourth power of $Z$, explain some of the forbidden lines in the solar corona.

More work needs to be done on the third and fourth spectra in the iron group to meet the needs of astronomers, but progress is being made with selected spectra in this group.

This review contains a current bibliography and an appraisal of the present state of analysis of individual spectra arranged by sequences. The spectra on which "work is in progress" are also indicated. He is preparing a similar report on spectra of the elements of the second half of the Periodic Table $(Z>30)$.

Current work on atomic spectra has been reported from several laboratories. At the Institute of Physics in Lund, B. Edlén lists the following spectra as being investigated: Be III, O II, F IV, F V, F vi, P III, P Iv, P v, S II, K Ix, Ca x, Sc II, Sc Iv, Sc v, Ti III, Ti Iv, Ti xII, V v, V vi, Fe Iv.

At the Lund Institute of Technology, L. Minnhagen and his associates have work in progress on O III, Mg III, Mg IV, Ar I, K II, Sr III, Sr IV, Ba III, Ba IV.

R. D. Cowan has noted several references to investigations of highly-ionized spectra of $\mathrm{V}, \mathrm{Cr}$, $\mathrm{Mn}$ and $\mathrm{Ni}$, and their significance in the interpretation of lines observed in the far ultraviolet solar spectrum and in solar flares $(5,7,8)$.

W. R. S. Garton and his colleagues have continued their work in the short-wave region. They have observed Rydberg series and autoionization resonances in the adsorption spectra of alkali and alkaline-earth atoms.

An annual report from the Spectroscopy Section of the National Bureau of Standards, prepared by L. B. Hagan is in press (29). Encouraging progress is being made on the analysis of rare-earth spectra. A general description of the present status of these spectra is described by W. C. Martin in two papers on the energy-level structures of lanthanide atoms and ions $(46,47)$. From 19 known energy differences of the type $4 f^{N-1} n l\left(6 s^{M}\right)-4 f^{N}\left(6 s^{M}\right)$ (with each configuration represented by its lowest level) he has predicted 23 values. "The resulting data confirm striking regularities among the $f$-shell graphs first used by Racah" to predict unknown system differences.

From absolute measurements in He II E. G. Kessler has determined the Rydberg constant to be $109737.3208 \pm 0.0085 \mathrm{~cm}^{-1}$ (41). 
E. Meinders has summarized the spectra on the programs of a number of laboratories, in her report of the Fourth Conference of the European Group of Atomic Spectroscopists, held at the Zeeman Laboratory in Amsterdam in 1972 (50).

Those interested in individual spectra are urged to consult a general "Bibliography on Atomic Energy Levels and Spectra" for the period July 1968 through June 1971, prepared by L. B. Hagan and W. C. Martin (30). This is a continuation of the 1968 Bibliography by C. E. Moore (53). A limited number of later references not included in this Bibliography are appended to the present report. A key indicating the reference numbers that apply to individual spectra, precedes the reference list.

Some general tables may, also, be of interest. A compilation of "Ionization Potentials and Ioniza tion Limits Derived from the Analyses of Optical Spectra" is available (54).

Progress is being made on the preparation of additional sections of the National Standard Reference Data Series on "Selected Tables of Atomic Spectra": Section 4, N IV to N vir and Section 6, $\mathrm{H}_{\mathrm{I}}, \mathrm{D}, \mathrm{T}$ are in print $(55,56)$. Manuscript has been completed for $\mathrm{N}$ I, $\mathrm{N}$ I, and He I, He I.

\section{Key to References}

\begin{tabular}{|c|c|c|c|c|c|}
\hline Spectrum & Reference & Spectrum & Reference & Spectrum & Reference \\
\hline \multirow[t]{2}{*}{ H I } & 56 & Mg III & 1 & $\operatorname{Ar} x$ & 23 \\
\hline & & $\mathrm{Mg}_{\mathrm{IV}}$ & 2,36 & $\operatorname{Ar} \mathbf{X I}$ & 23 \\
\hline \multirow[t]{2}{*}{ He II } & 41 & $\mathrm{Mg}$ VII & 19 & Ar XII & 23 \\
\hline & & $\mathrm{Mg} \mathrm{X}$ & 19 & Ar XIV & 23 \\
\hline $\mathrm{Be} \mathrm{I}$ & 35 & & & $\operatorname{Ar} x v$ & 23 \\
\hline $\mathrm{Be}$ II & 35 & $\mathrm{Al}$ VIII & 19 & & \\
\hline \multirow[t]{2}{*}{ Be III } & 11 & Al IX & 42 & K VIII & 12 \\
\hline & & $\mathrm{Al} \mathrm{x}$ & 19,42 & K XIII & 3 \\
\hline \multirow[t]{2}{*}{ B I } & 27 & Al $\mathbf{x I}$ & & $\mathrm{K}$ xIV & 3 \\
\hline & & & & $\mathrm{K} \mathrm{xV}$ & 3 \\
\hline $\mathrm{N}_{\mathrm{I}}$ & 17 & Si VIII & 23,38 & & \\
\hline N IV & 55 & Si IX & $19,23,38$ & Ca IX & 12 \\
\hline $\mathrm{Nv}$ & 55 & Si $x$ & 23 & Ca XIV & 18 \\
\hline $\mathrm{N} \mathrm{vI}$ & 55 & Si XIr & 19 & Ca Xv & 18 \\
\hline \multirow{2}{*}{ N VII } & 55 & & & & \\
\hline & & P VIII & 40 & Sc I & 26 \\
\hline \multirow[t]{2}{*}{ O VII } & 16 & P IX & 40 & $\mathrm{Sc}$ II & 66 \\
\hline & & $\mathbf{P x}$ & 40 & $\mathrm{Se}$ III & 34 \\
\hline$F v$ & 19 & $\mathbf{P X I}$ & 40 & Sc VI & 21 \\
\hline F VI & 19 & P xII & 40 & Sc VII & 21 \\
\hline F vir & 19 & P XIII & 40 & Sc VIII & 21 \\
\hline \multirow[t]{2}{*}{ F VIII } & 16 & & & Sc $x$ & 12 \\
\hline & & $\mathrm{S}_{\mathrm{I}}$ & 72 & & \\
\hline Ne I & 39 & S IX & 23,60 & Sc XIII & 18 \\
\hline $\mathrm{Ne}$ II & 58 & $\mathrm{Sx}$ & 23,60 & Sc XIV & 18 \\
\hline Ne III & 45 & S XI & $23,38,60$ & Sc Xv & 18 \\
\hline $\mathrm{Ne}$ IV & 44 & S XII & 23,60 & & \\
\hline $\mathrm{Nev}$ & 44 & & & Tì II & 66 \\
\hline $\mathrm{Ne}$ vI & 44 & $\mathrm{Clx}$ & 23 & Ti vI & 21 \\
\hline $\mathrm{Ne}$ vII & 44 & $\mathrm{Cl} \mathrm{XI}$ & 23 & Ti VII & 21,70 \\
\hline Ne vill & 9,57 & $\mathrm{Cl}$ XII & 23 & Ti VIII & 21 \\
\hline \multirow[t]{2}{*}{$\mathrm{Ne} I \mathrm{X}$} & 9,57 & $\mathrm{Cl}$ XIII & 23 & Ti IX & 21 \\
\hline & & $\mathrm{Cl}$ xIV & 23 & Ti XI & 12,21 \\
\hline $\mathrm{Na}$ III & 52 & & & Ti XIV & 18 \\
\hline $\mathrm{Na}$ vI & 19 & Ar I & $31^{*}$ & Ti xv & 18 \\
\hline \multirow{2}{*}{ Na $1 x$} & 19 & Ar II & 51 & & \\
\hline & & Ar VIII & 6,9 & & \\
\hline
\end{tabular}


Key to References (continued)

\begin{tabular}{|c|c|c|c|c|c|}
\hline Spectrum & Reference & Spectrum & Reference & Spectrum & Reference \\
\hline $\begin{array}{l}\text { Vd II } \\
\text { Vd VII }\end{array}$ & $\begin{array}{l}66 \\
21\end{array}$ & $\begin{array}{l}\text { Mn II } \\
M n I X\end{array}$ & $\begin{array}{l}66 \\
22\end{array}$ & Zn I & 48 \\
\hline Vd VIII & 21 & Mn $x$ & 20,22 & As II & 43 \\
\hline Vd ix & 21 & $\operatorname{Mn} \mathrm{XI}$ & 20,22 & & \\
\hline $\operatorname{Vdx}$ & 21 & Mn XII & $20,21,22$ & Kr I & 31* \\
\hline $\mathrm{VdxI}$ & 22 & Mn XIII & $20,21,22$ & & \\
\hline Vd xu & 21,22 & Mn XIV & 20,22 & Rb Ir & 64 \\
\hline Vd XIII & 22 & Mn xv & 22 & Rb III & 33,63 \\
\hline \multirow[t]{2}{*}{$V d x v$} & 18 & & & & \\
\hline & & Fe II & 66 & Sr III & 59,64 \\
\hline Cr II & 66 & $\mathrm{Fe}$ IX & 22 & & \\
\hline Cr viu & 20 & Fe $x$ & $20,22,74$ & $Y t I$ & 25 \\
\hline Cr IX & 20,21 & Fe XI & $20,22,38,74$ & Yt IV & 64 \\
\hline $\operatorname{Cr} x$ & 20,21 & Fe XII & $20,22,38,74$ & Yt v & 62 \\
\hline Cr XI & 20,21 & Fe XIII & $20,22,38,74$ & & \\
\hline Cr XII & 20,22 & Fe XIV & $20,22,74$ & $\mathrm{Zr} \mathrm{v}$ & 64 \\
\hline Cr xIII & 21,22 & $\mathrm{Fe} x \mathrm{v}$ & $5,20,21,22$ & Zr vi & 13 \\
\hline $\mathrm{Cr} \mathrm{XIV}$ & 22 & Fe XVI & 22 & & \\
\hline \multirow[t]{22}{*}{$\mathrm{Cr} \times \mathrm{xv}$} & 71 & & & $\mathrm{Nb}$ vi & 64 \\
\hline & & Co II & 66 & $\mathrm{Nb}$ vir & 14 \\
\hline & & $\operatorname{Cox}$ & 21,24 & & \\
\hline & & Co XI & 21,24 & Mo vil & 64 \\
\hline & & Co XII & 21,24 & Mo VIII & 15 \\
\hline & & Co XIII & 21,24 & & \\
\hline & & Co XIV & 21,24 & $\mathrm{Cd} \mathrm{I}$ & 48 \\
\hline & & $\operatorname{Coxv}$ & 21,24 & & \\
\hline & & Co XvI & 21,24 & La III & 37 \\
\hline & & Co xvII & 21,24 & La IV & 32 \\
\hline & & Ni it & $65,66,67$ & Ce I & 73 \\
\hline & & $\mathrm{Ni} \mathrm{xI}$ & 21 & Ce II & 73 \\
\hline & & Ni XII & 21 & $\mathrm{CeV}$ & 61 \\
\hline & & Ni XIII & 21,38 & Ce IV & 61 \\
\hline & & $\mathrm{Ni}$ XIV & 21,38 & Dy I & 28 \\
\hline & & $\mathrm{Nixv}$ & 21,38 & $\operatorname{Tm} \mathrm{I}$ & 69 \\
\hline & & $\mathrm{Ni}$ xvi & 21 & Tm II & 4 \\
\hline & & Ni xvil & 21,74 & Lu iv & 68 \\
\hline & & Ni xvIII & 21,74 & $\mathbf{H g}$ & 48,49 \\
\hline & & & & $\mathrm{Tl}$ II & 49 \\
\hline & & & & Pb III & 49 \\
\hline & & & & Bi IV & 49 \\
\hline
\end{tabular}

* Refers to isoelectronic sequence

\section{REFERENCES}

1. Andersson, E., Johannesson, G. A. 1971, Phys. Scr., 3, 203.

2. Artru, M.-C., Kaufman, V. 1972, J. Opt, Soc. Am., 62, 949.

3. Boiko, V. A., Voinov, Yu. P., Gribkov, V. A., Sklizkov, G. V. $\quad$ 1970, Opt. Spectrosc., 29, 545.

4. Camus, P., Sugar, J. 1971, Phys. Scr., 4, 257, 263.

5. Cowan, R. D., Widing, K. G. 1973, Astrophys. J., 180, 285.

6. Datla, R. U., Kunze, H.-J., Petrini, D. 1972, Phys. Rev., 6A, 38.

7. Doschek, G. A., Meekins, J. F., Cowan, R. D. 1972, Astrophys. J., 177, 261.

8. Doschek, G. A., Meekins, J. F., Cowan, R. D. 1973, Solar Phys., in press.

9. Druetta, M. Datla, R. U., Kunze, H. -J. 1972, Astrophys. J., 174, 215.

10. Edlén, B. 1972, Meeting, European Group for Atomic Spectroscopy. 
11. Eidelsberg, M. 1972, J. Phys. B: At. Molec. Phys., 5, 1031.

12. Ekberg, J. O. 1971, Phys. Scr., 4, 101.

13. Ekberg, J. O., Hansen, J. E., Reader, J. 1972, J. Opt. Soc. Am. 62, 1134.

14. Ekberg, J. O., Hansen, J. E., Reader, J. 1972, J. Opt. Soc. Am., 62, 1139.

15. Ekberg, J. O., Hansen, J. E., Reader, J. 1972, J. Opt. Soc. Am., 62, 1143.

16. Engelhardt, W., Sommer, J. 1971, Astrophys. J., 167, 201.

17. Eriksson, K. B. S., Petersson, J. E. 1971, Phys. Scr., 3, 211.

18. Fawcett, B. C. 1971, J. Phys. B: At. Molec. Phys., 4, 981.

19. Fawcett, B. C. 1971, J. Phys. B: At. Molec. Phys., 4, 1115.

20. Fawcett, B. C. 1971, J. Phys. B: At. Molec. Phys., 4, 1577.

21. Fawcett, B. C., Cowan, R. D., Hayes, R. W. 1972, J. Phys. B: At. Molec. Phys., 2143.

22. Fawcett, B. C. ,Cowan, R. D., Kononov, E. Ya., Hayes, R. W. 1972, J. Phys. B: At. Molec. Phys., $5,1255$.

23. Fawcett, B. C., Gabriel, A. H., Paget, T. M. 1971, J. Phys. B: At. Molec. Phys., 4, 986.

24. Fawcett, B. C., Hayes, R. W. 1972, J. Phys. B: At. Molec. Phys., 5, 366.

25. Garton, W. R. S., Reeves, E. M., Tomkins, F. S., Ercoli, B. $\quad$ 1972, Proc. R. Soc. (London), in press.

26. Garton, W. R. S., Reeves, E. M., Tomkins, F. S., Ercoli, B. 1972, Proc. R. Soc. (London), in press.

27. Goorvitch, D., Valero, F. P. J. 1972, Astrophys. J., 171, 643.

28. Griffin, D. C., Ross, J. S., Cowan, R. D. 1972 J. Opt. Soc. Am., 62, 571.

29. Hagan, L. B. 1973, Bull. Am. Astron. Soc., in press.

30. Hagan, L. B., Martin, W. C. 1972, Nat. Bur. Std. (U.S.) Spec. Publ., 363.

31. Hansen, J. E. 1972, J. Phys. B: At. Molec. Phys., 5, 1083.

32. Hansen, J. E. 1972, J. Phys. B: At. Molec. Phys., 5, 1096.

33. Hansen, J. E., Persson W., Valend, S. 1972, Phys. Lett., in press.

34. Holmstrom, J.-E. 1972, Phys. Scr., in press.

35. Hontzeas, S., Martinson, I., Erna, P., Buchta, R. 1972, Phys. Scr., in press.

36. Johannesson, G. -A., Lundstrom, T. 1972, Phys. Scr., in press.

37. Johannesson, S., Litzen, U. 1971, J. Opt. Soc. Am., 61, 1427.

38. Jordan C. 1971, Solar Physics, 21, 381.

39. Kaufman, V., Minnhagen, L. $1972, J$. Opt. Soc. Am., 62, 92.

40. Kasynnov, Yu. S., Kononov, E. Ya., Korobkin, V. V., Koshelev, K. N., Serov, R. V. $\quad 1972$, Opt Spectrosc., in press.

41. Kessler Jr., E. G. 1973, Phys. Rev. A.

42. Kononov, E. Ya., Koshelev, K. N., Ryabtsev, A. N. 1971, Opt. Spectrosc., 30, 534.

43. Li, H., Andrew, K. L. 1972 , J. Opt. Soc. Am., 62, 255.

44. Lindeberg, S. 1972, Upsala Univ. Inst. Phys., UUIP- 758, 759, 760.

45. Lundstrom, T., Minnhagen, L. 1972, Phys. Scr., in press.

46. Martin, W. C. 1971, J. Opt. Soc. Am., 61, 1682.

47. Martin, W. C. 1972 , Opt. Pura. Apl., in press.

48. Martin, W. C., Sugar, J., Tech, J. L. 1972, J. Opt. Soc. Am., 62, 1488.

49. Martin, W. C., Sugar, J., Tech, J. L. 1972, Phys. Rev., A6, 2002.

50. Meinders, E. 1972, Eur. Group At. Spectrosc.

51. Minnhagen, L. 1971, J. Opt. Soc. Am., 61, 1257.

52. Minnhagen, L., Nietsche, H. 1972 , Phys. Scr., in press.

53. Moore, C. E. $\quad 1968,1969$, Nat. Bur. Std. (U.S.) Spec. Publ. 306, 4 sections.

54. Moore, C. E. $\quad 1970$, Nat. Stand. Ref. Data Ser. Nat. Bur. Std. (U.S.), 34.

55. Moore, C. E. $\quad 1971$, Nat. Stand. Ref. Data Ser., Nat. Bur. Std. (U.S.) 3, Sec. 4.

56. Moore, C. E. 1972, Nat. Stand. Ref. Data Ser., Nat. Bur. Std. (U.S.), 3, Sec. 6.

57. Peacock, N. J., Speer, R. J., Hobby, M. G. $\quad$ 1969, J. Phys. B: At. Molec. Phys., 2, 798.

58. Persson, W. 1971, Phys. Scr., 3, 133.

59. Persson, W., Valind, S. 1972 , Phys. Scr., in press.

60. Podobedova, L. I., Kononov, E. Ya., Koshelev, K. N. 1971, Opt. Spectrosc., 30, 217.

61. Reader, J., Ekberg, J. O. 1972, J. Opt. Soc. Am., 62, 464.

62. Reader, J., Epstein, G. L. $\quad$ 1972, J. Opt. Soc. Am., 62, 619.

63. Reader, J., Epstein, G. L. $\quad 1972$, J. Opt. Soc. Am., 62, 1467.

64. Reader, J., Epstein, G. L., Ekberg, J. O. 1972, J. Opt. Soc. Am., 62, 273.

65. Shadmi, Y., Caspi, E. $\quad 1972$, J. Res. Nat. Bur. Std. (U.S.), 76A, 125.

66. Shadmi, Y., Oreg, J., Stein, J. $\quad$ 1968, J. Opt. Soc. Am., 58, 909. 
67. Shenstone, A. G. $\quad 1970,1971$, J. Res. Nat. Bur. Std. (U.S.), 74A, 801; 75A, 335.

68. Sugar, J., Kaufman, V. 1972, J. Opt. Soc. Am., 62, 562.

69. Sugar, J., Meggers, W. F., Camus, P. 1972, J. Res. Nat. Bur. Std. (U.S.), 76A, in press.

70. Svensson, L. A. 1971, Phys. Scr., 4, 111.

71. Swartz, M., Kastner, S., Rothe, E., Neupert, W. 1971, J. Phys. B: At. Molec. Phys., 4, 1747.

72. Tondello, G. 1972, Astrophys. J., 172, 771.

73. Verges, J., Corliss, C. H., Martin, W. C. 1972, J. Res. Nat. Bur. Std. (U.S.), 76A, 285.

74. Widing, K. G., Sandlin, G. D., Cowan, R. D. 1971, Astrophys. J., 169, 405.

CH. MOORE-SITTERLY

Chairman of the Committee

\section{COMMITTEE 5: MOLECULAR SPECTRA}

\section{Compendia, bibliographies and atlases}

The period of time covered by this report saw the appearance of a number of important publications. Of primary importance has been the appearance of the second edition of Spectroscopic Data relative to Diatomic Molecules (1) preprared by an international team of spectroscopists under the direction of B. Rosen. About 700 diatomic molecules are covered. For each molecule most of the following are included: mode of production, wavelengths and classification of vibrational band heads, molecular constants, dissociation energies, perturbations, predissociations, ionization potentials, transition probabilities, and a detailed bibliography to 1969. As a follow-up to this enormous task, a group is preparing to produce annual critical abstracts and up-dates. A program is being written at Stockholm to catalogue data from this table, both by molecule and by wavelength. Several bibliographies have appeared or are in preparation. Carlos Suarez at La Plata has compiled via computer a Bibliography of Spectra of Diatomic Molecules, 1960-1970. It is in four parts; a few polyatomic molecules are also listed. The International Union of Pure Applied Chemistry publishes a Bibliography on the High Temperature Chemistry and Physics (2) which includes many interesting references to laboratory techniques, specific heats, transition probabilities, etc. T. M. Dunn at Michigan and T. Larsson and H. Neuhaus at Stockholm are maintaining bibliographies of hyperfine structures in diatomic molecules, David R. Lide of the Office of Standard Reference Data, N.B.S., has called attention to the new Journal of Physical and Chemical Reference Data, which will include compilations and reviews of interest to molecular spectroscopists. The bi-monthly Berkeley News letters are continuing; the mailing list has grown to cover 400 spectroscopists in various countries. Each bi-monthly issue includes a bibliography of papers on diatomic and simpler polyatomic molecules appearing in over 30 journals. R. W. Nicholls has continued the publication of the series of Identification Atlases of Molecular Spectra, the most recent covering the CO Fourth Positive System, VO, and the CN Red System; an atlas on the CN violet system is in preparation. Publication of the CRESS reports by Nicholls and his collaborators has continued; they include data on FranckCondon factors and related quantities for a long series of band systems. Lifetimes in gaseous diatomic molecules have been discussed in a recent article by Anderson (3). Finally a number of monographs of great value have appeared. They include: Rotational Structure in the Spectra of Diatomic Molecules by I. Kovacs (4), Far-infrared Spectroscopy by Moller and Rothschild (5), Microwave Molecular Spectra by Gordy and Cook (6), Rydberg series in Atoms and Molecules by Duncan (7), An Introduction to Molecular Spectroscopy by Herzberg (8), Low Temperature Spectroscopy by B. Meyer (9) and Revised Tables of Diatomic Molecular Constants by K. P. Huber (10).

\section{Molecular data}

Even if the discussion is limited to molecules of astrophysical interest, the research that has been completed is much too extensive for full coverage here. Reference should be made to the bibliographies listed above. A selection of recent analyses includes the following. 